\title{
Development and Evaluation of a Simple Algorithm to Find Cloud Optical Depth with Emphasis on Thin Ice Clouds
}

\author{
J.C. Barnard ${ }^{*}, \mathrm{a}$, C.N. Long ${ }^{\mathrm{a}}$, E.I. Kassianov ${ }^{\mathrm{a}}$, S.A. McFarlane ${ }^{\mathrm{a}}$, J.M. Comstock ${ }^{\mathrm{a}}$, M. Freer ${ }^{\mathrm{b}}$ and \\ G.M. McFarquhar ${ }^{\mathrm{b}}$
}

${ }^{a}$ Pacific Northwest National Laboratory, Richland, Washington, USA

${ }^{b}$ University of Illinois at Urbana-Champaign, Urbana, Illinois, USA

\begin{abstract}
An algorithm is presented here for determining cloud optical depth, $\tau$, using data from shortwave broadband irradiances, focusing on the case of optically thin clouds. This method is empirical and consists of a one-line equation. This method is applied to cirrus clouds observed at the Atmospheric Radiation Measurement Program Climate Research Facility (ACRF) at Darwin, Australia, during the Tropical Warm Pool International Cloud Experiment (TWP-ICE) campaign and cirrus clouds observed at the ACRF Southern Great Plains (SGP) site. These cases were chosen because independent verification of cloud optical depth retrievals was possible. For the TWP-ICE case, the calculated optical depths agree to within 1 unit with $\tau$ calculated from a vertical profile of ice particle size distributions obtained from an aircraft sounding. For the SGP case, the results from the algorithm correspond reasonably well with $\tau$ values obtained from an average over other retrieval methods, some of which have been subject to independent verification. The medians of the two time series are 0.79 and 0.81 , for the empirical and averaged values, respectively. Because such close agreement is likely to be fortuitous and therefore not truly represent the performance of our method, $\tau$ values derived from our method were compared to values obtained from lidar data. Over a three year period, the difference in median values between the two methods is about 0.6 , with the lidar optical depths being larger. This tool may be applied wherever measurements of the direct, diffuse, and total components of the shortwave broadband flux are available at 1- to 5-minute resolution. Because these measurements are made across the world, it then becomes possible to estimate optical depth for both liquid water and ice clouds at many locations.
\end{abstract}

Keywords: Cloud, optical depth, algorithm.

\section{INTRODUCTION}

Optically thin clouds are now an important topic in the climate community because of their ubiquity and effect on radiative fluxes. The defining feature of such clouds is their low water (ice) path and correspondingly low optical depth. Accurate determination of these and other cloud properties is critical for their representation in climate models, but the "thinness" of these clouds makes it difficult to determine their microphysical and optical properties, particularly using remote sensing [1].

Remote sensing of cloud visible optical depth, $\tau$, is possible using measurements from a variety of instruments along with their respective retrieval algorithms, as described in Turner et al. [1] for thin liquid water clouds, and Comstock et al. [2] for ice clouds. To this myriad mix of algorithms, we present here yet another algorithm that uses data from broadband radiometers to find $\tau$. This method is simple to use and requires minimal amounts of computer time. Considering these factors and the relative abundance of broadband irradiance measurements compared to more sophisticated and expensive instruments, the algorithm may be used to easily estimate thin cloud properties at many locations worldwide.

*Address correspondence to this author at the Pacific Northwest National Laboratory, Richland, Washington, USA; E-mail: james.barnard@pnl.gov
In a previous paper [3], abbreviated henceforth as BL, we described the development of the algorithm as applied to optically thick water clouds. Here we derive a more elegant and physically based formulation. Although this new formulation is strictly speaking valid only for thick clouds, we apply the method to the optically thin case in hopes that it works reasonably well in comparison to other, more sophisticated algorithms. Such hopes are borne out, as described below.

\section{ALGORITHM DEVELOPMENT AND ALGORITHM ERRORS}

\subsection{Algorithm Development}

The original algorithm described in BL is entirely empirical and uses broadband irradiances to find $\tau$. In preparation for the derivation of the more physically based algorithm used in this paper, we briefly summarize the empirical derivation presented in BL. The basic underpinning of the empirical formula presented in BL is that, for a horizontally homogenous, optically thick cloud deck, $\tau$, should be a function of the diffuse irradiance transmission and the cosine of the solar zenith angle, $\mu_{0}$. The diffuse transmission is defined here as $D / C$, where $D$ is the measured diffuse irradiance and $C$ is the clear sky total shortwave irradiance, the shortwave irradiance that would be measured in the absence of clouds. This quantity is found easily using the shortwave flux analysis described in [4] and has been used in rigorous broadband 
algorithms, which use detailed radiative transfer calculations to find $\tau$ [5]. In the original formulation, we postulated that

$\tau_{\text {empirical }}=f\left(D / C \mu_{0}^{\alpha}\right)$,

where $\tau_{\text {empirical }}$ is an empirical representation of $\tau, \alpha$ is an exponent, to be determined, and $f$ is a functional form, also to be determined. We found $\alpha$ by first plotting $\tau$ versus the variable, $r=D /\left(C \mu_{0}{ }^{\alpha}\right)$, for a given value of $\alpha$. Then using a trial-and-error procedure $\alpha$ was adjusted repeatedly to achieve the best possible "collapse" of the $\tau$ values on a single curve so that $\tau$ was, to the extent possible, a single valued function of $r$.

This procedure requires $\tau$ values and these were generated using the algorithm of Min and Harrison [6], which calculates $\tau$ for optically thick ( $\tau \geq 5$ ), plane parallel, liquid water clouds using diffuse irradiance data from the Multi-Filter Rotating Shadowband Radiometer (MFRSR) [7]. The result of this procedure is shown in Fig. (1) (similar to Fig. (1) in BL). This plot shows $\log [\tau]$, where $\tau$ is calculated using the Min and Harrison algorithm, plotted versus $r$. The irradiance data

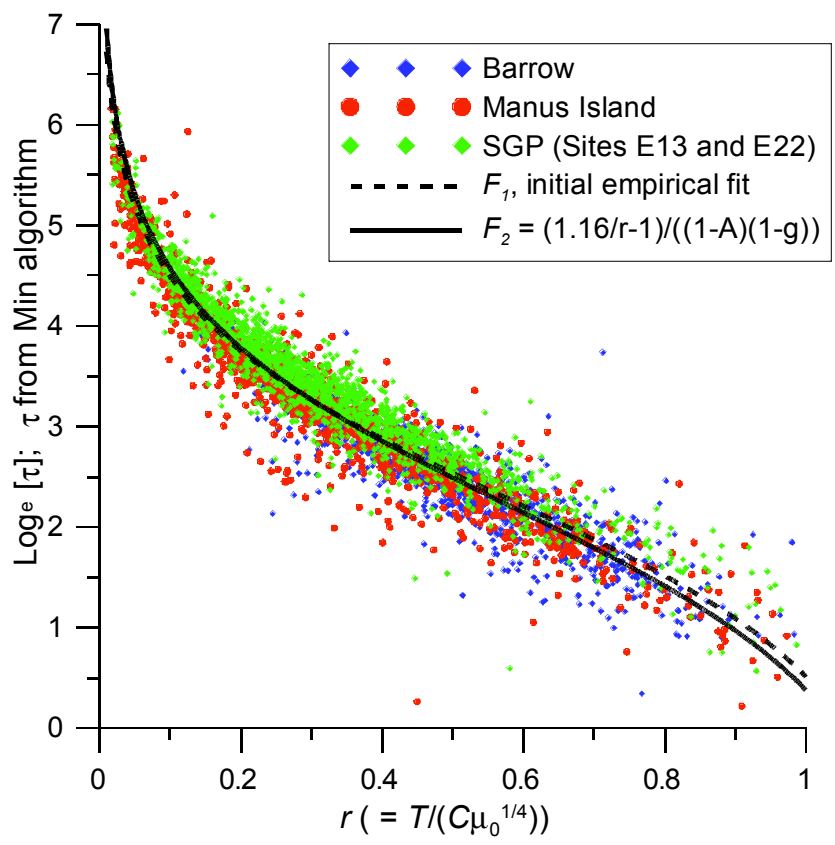

Fig. (1). Equation (9) as fitted to cloud optical depth calculations. This figure also shows the function $F_{l}$, the initial empirical fit from BL.

required to determine $\tau$ were obtained from the ACRF site in Barrow, Alaska, the ACRF Tropical Western Pacific (TWP) Manus Island site, and two ACRF SGP sites. The number of points used in this analysis, representing 5-min averages of $r$ and $\tau$ over the year 2000, ranges from about 6700 at Barrow to about 9100 for the two SGP sites. To remove the effect of broken cloudiness that acutely violates the plane parallel assumption required by the Min algorithm, we only considered cases in which the fractional sky cover, as determined by applying the Long algorithm [8] to broadband irradiances, was greater than 0.99 . The plot shows the best "collapse" of the data which occurs when the exponent $\alpha$ was chosen to be $1 / 4$. Further justification for this choice of exponent will be provided below. A hyperbolic arctangent was chosen as the function $f$ in Eq. (1), and this function is shown in Fig. (1). We denote this particular function $f$ as $F_{I}$.

In contrast to this completely empirical approach, our new algorithm is partially based on the physics of radiative transfer, and can therefore be described as semi-empirical. The derivation of the new algorithm begins with the Eddington approximation described by Shettle and Weinman [9]. In this approximation, the total irradiance, $T$, at the Earth's surface is:

$$
\begin{aligned}
& T=\mu_{0}(\pi F) \\
& \left(e^{-\tau / \mu_{0}}+\frac{\left(\left(2+3 \mu_{0}\right)\left(1-e^{-\tau / \mu_{0}}\right)-e^{-\tau / \mu_{0}} 3(1-A)(1-g) \tau\right)}{(4+3(1-A)(1-g) \tau)},\right.
\end{aligned}
$$

where $\pi F\left(\mathrm{~W} / \mathrm{m}^{2}\right)$ is an extraterrestrial irradiance; $A$, is the broadband surface albedo; and $g$ is the cloud droplet or ice particle asymmetry factor. For our derivation, we assume that clouds are the only agents that act on the solar radiation, and that there is no cloud absorption. As noted by $\mathrm{Hu}$ and Stamnes [10] this latter assumption is good for the visible region of the solar spectrum. For large $\tau / \mu_{0}(\geq 5), T$ becomes

$T=(\pi F) \frac{\mu_{0}\left(2+3 \mu_{0}\right)}{(4+3(1-A)(1-g) \tau)}$.

We now re-define the parameter, $r$, as

$r=\frac{T}{C \mu_{0}^{\alpha}}$,

where $T\left(\mathrm{~W} / \mathrm{m}^{2}\right)$ is the measured total irradiance. Note that we now use the measured total irradiance, $T$, whereas the old algorithm used the measured diffuse irradiance, $D$. As noted in Long and Ackerman [4], the diurnal variation of $C$ is most dependent on $\mu_{0}$; this dependence is well-represented by the empirical fit, $C=B \mu_{0}^{1.25}$, where $B\left(\mathrm{~W} / \mathrm{m}^{2}\right)$ is a fitting constant. The variable $r$ is therefore

$r=\frac{(\pi F)}{B \mu_{0}^{1.25}} \frac{\mu_{0}\left(2+3 \mu_{0}\right)}{(4+3(1-A)(1-g) \tau) \mu_{0}^{\alpha}}$.

The numerator $\mu_{0}\left(2+3 \mu_{0}\right)$ is approximated extremely well by $5 \mu_{0}^{1.51}$ and making this substitution yields

$r=$

$\frac{(\pi F)}{B \mu_{0}^{1.25}} \frac{5 \mu_{0}^{1.51}}{(4+3(1-A)(1-g) \tau) \mu_{0}^{\alpha}}$

$=\frac{(\pi F)}{B} \frac{5}{(4+3(1-A)(1-g) \tau)}\left(\frac{\mu_{0}^{1.51}}{\mu_{0}^{1.25} \mu_{0}^{\alpha}}\right)$

Taking $\alpha=1 / 4$ effectively removes the dependence on the solar zenith angle on the right hand side of Eq. (6). Solving for $\tau$ gives 


$$
\tau=\frac{\frac{4}{3}\left(\frac{5 \pi F / B}{4 r}-1\right)}{(1-A)(1-g)},
$$

which suggests an empirical relationship of the form

$$
\tau=\frac{\left(\frac{c_{1}}{r}-1\right)}{(1-A)(1-g)},
$$

where $c_{l}$ is a fitting constant. This constant is determined by trial and error: it is adjusted until the best fit between the optical depth from the Min algorithm and the empirical equation, Eq. (8), is achieved. The best fit, assessed visually, is given with $c_{1} \approx 1.16$ and the expression for the empirical optical depth, $\tau_{\text {empirical }}$, is therefore

$\tau=\tau_{\text {empirical }}=F_{2}(r)=\frac{\left(\frac{1.16}{r}-1\right)}{(1-A)(1-g)}$.

This equation, denoted as $F_{2}(r)$, is also shown in Fig. (1), where it is seen that $F_{l}(r)$, the hyperbolic arctangent, and $F_{2}(r)$ are nearly identical. Therefore the application of Eq. (9) to optically thick liquid water clouds should produce the same values of $\tau_{\text {empirical }}$ as discussed in BL, and we will not discuss the case of optically thick clouds here. Rather we focus on optically thin ice clouds.

\subsection{Errors}

The parameter $r=(T / C) / \mu_{0}^{1 / 4}$ in Eq. (9) is proportional to the atmospheric transmission $(T / C)$ throughout the entire atmospheric column that includes clouds and clear air. Using this definition, the transmission is defined with respect to the clear sky irradiance, $C$, rather than the top of atmosphere irradiance. (Note also that because $T$ and $C$ are obtained from the same set of instruments, calibration errors cancel in the ratio.) For the cloudy skies of interest, $C$ is an estimate of the hypothetical clear sky irradiance, defined as the irradiance that would be measured in the absence of the clouds. Using $C$ to define transmission tends to reduce retrieval errors because factors that affect transmission in the clear air above and below the clouds are approximately accounted for.

These factors include aerosol optical depth, $\tau_{\text {aer }}$; abundances of water vapor and ozone, and surface pressure. Quantitative error estimates caused by variations in these factors are provided in BL. Here we briefly explain why the errors associated with these factors tend to be small. The clear sky irradiance, $C$, is found by the realization that the surface solar fluxes are most influenced by $\mu_{0}$, and accordingly, $C$ is assumed to have the form $C \approx B \mu_{0}^{b}$, where $B$ and $b$ are fitting parameters. These parameters are chosen to minimize the difference between $B \mu_{0}^{b}$ and the measured clear sky, total downwelling shortwave irradiance during the day. This procedure finds a $B$ and $b$ for each clear day in a long time series of irradiance data; typically $b$ is about 1.25 while $B$ is about $1100 \mathrm{~W} / \mathrm{m}^{2}$. For days that do not meet the clear sky criterion, interpolation of the parameters $B$ and $b$ for clear days on either side of the day in question is used.
The resulting estimate for the clear sky total downwelling irradiance therefore contains some information about the background aerosol, water vapor abundances, and variations in surface pressure. Long and Ackerman [4] tested this procedure by examining the irradiances calculated for a clear day using $B$ and $b$ interpolated from other clear days before and after this day. They found that the root mean square error of the "interpolated" irradiances versus the measurements was about $13 \mathrm{~W} / \mathrm{m}^{2}$, within the measurement error of total irradiance. This suggests that, in most cases, variations in $\tau_{a e r}$, water vapor, and surface pressure will be mostly accounted for in the calculation of the transmission, because the value of $C$ for the day in question is likely to be a good estimate of the true, clear sky surface irradiance. Rare exceptions to this conclusion would occur, for example, during pollution events with very large values of $\tau_{a e r}$, far outside the typical range. Such atypically large values would not be captured by the procedure described above.

Cloud properties such as effective particle radius and particle shape affect our results. In the old formulation given in $\mathrm{BL}$, these properties were not explicitly taken into account. By contrast, in the new method these factors are approximately dealt with in the choice of $g$ in Eq. (9); this will be discussed in section 4 . To avoid problems associated with mixed phase clouds, we focus exclusively on ice clouds in this study. These clouds occur at very large altitudes with correspondingly cold temperatures so that liquid water cannot exist. To avoid the problem of broken cloud scenes, we use the algorithm of Long et al. [8] to identify cases when the fractional sky cover is greater than 0.9. Even in the presence of fractional sky covers in excess of 0.9 , the clouds will not likely be horizontally and vertically homogenous. In this regard, the optical depth so retrieved should be considered an "effective" optical depth, meaning that it is some average over the clouds within the field of view of the instruments measuring the shortwave irradiances.

Because the focus here is on thin ice clouds we cannot overemphasize that Eq. (9) was derived for the optically thick case (defined here as $\tau>5$ ), and there is no rigorous physical reason to expect it to work for the thin cloud case. Indeed, in the asymptotic limit of clear skies, such that $T \approx$ $C$, Eq. (9) yields $\tau_{\text {empirical }}$ values between about -1.4 to about 1.0 , depending upon the solar zenith angle, although the averaged value of $\tau$ for $0.2 \leq \mu_{0} \leq 1.0$ is virtually zero. (The value of $\mu_{0}$ at which $\tau_{\text {empirical }}$ equals zero is about 0.55 .) Cognizant of these problems, we nonetheless apply the algorithm to the thin cloud case, and we find that it works surprisingly well vis-à-vis other methods, particularly when finding distributions of optical depth. Given that at the present time the uncertainties associated with other retrieval methods are not well known, we think this method would be an excellent choice for predicting "effective" optical depth distributions over a wide range of optical depth.

\section{PHILOSOPHICAL CONSIDERATIONS: ALGO- RITHM VALIDATION}

Algorithm validation is only credible when the quantity retrieved by the algorithm can be verified in an independent 
manner. For cloud optical depth, this independent verification can be done in several ways, none of which is perfect. We mention two methods here. The first of these consists of deriving an optical depth based on in situ cloud measurements of particle size distributions and, in the case of ice clouds, ice particle shape. Obviously, an optical depth determined in this manner is completely independent from ground-based retrievals of $\tau$, and a comparison between the two provides some information of how well the retrieval algorithm works. The second method is termed "radiative flux closure". With this scheme, retrieved cloud properties such as $\tau$, asymmetry parameter, $g$, and single scattering albedo, $\varpi_{0}$, are fed to a radiative transfer model to calculate surface fluxes. The extent to which calculated and measured fluxes agree is used to evaluate the retrieval algorithm.

\subsection{Algorithm Validation Using a "First Principles" Cloud Optical Depth}

Goody and Yang [11] define the optical path as the integral of extinction between two points, $\mathrm{A}$ and $\mathrm{B}$, as

$$
\text { optical path }_{\lambda}=\int_{p o \text { int } B}^{p o \text { int } A} e_{v, \lambda}(s) d s,
$$

where $e_{v, \lambda}$ is the volume extinction coefficient with units (1/length), as a function of wavelength, $\lambda$; and ds is an element of path length between point $\mathrm{A}$ and point $\mathrm{B}$. The "normal" optical depth [12] of the cloud is the optical path found on a vertical line between the top and bottom of the cloud. We use this as our definition of cloud optical depth, and give it the label $\tau_{f p}$, where the subscript " $f p$ " stands for "first principles". Of course, $\tau_{f p}$ is a function of $\lambda$, but in the visible spectral region, it is not expected to vary significantly over wavelength [10] because ice crystals with radii between 10 and $1000 \mu \mathrm{m}$ have size parameters $(x=2 \pi r / \lambda)$ between 125 (21) and $12500(2100)$ for $\lambda=0.5(3.0 \mu \mathrm{m})$, suggesting geometric optics with an extinction coefficient of 2 applies over this spectral region.

In practice it is not possible to find $\tau_{f p}$ exactly, because the measurements needed to find $\tau_{f p}$ are subject to significant uncertainties. Here we estimate it using the profile of extinction between cloud top and cloud bottom. This profile can be determined from aircraft measurements of cloud particle size distribution, and cloud particle shape and phase throughout the depth of a cloud, although both horizontal inhomogeneities, and uncertainties and approximations in the observations complicate the analysis $[13,14]$. Once the size distribution, shape, and phase are known, Mie theory, or other computation techniques for non-spherical particles (e.g., ray tracing), can be applied to find $e_{v, \lambda}(z)$. Integration over the depth of the cloud yields an estimate of $\tau_{f p}$. To explicitly distinguish between the idealized, "first principle" optical depth and its estimate, we assign the symbol $\tau_{\text {est }}$ to this quantity.

When using in situ cloud measurements for the evaluation of algorithms, one must be mindful of the many considerations summarized by Comstock et al. [2]. These include: collocation errors and sampling volume inconsistencies be- tween the aircraft and the ground-based instruments, particle breakup [14] and other instrumental errors, and the inevitable inexactness of converting the measured data to extinction values caused, for example, by using idealized particle shapes to calculate extinction.

\subsection{Flux Closure Validation}

For completely clear skies, flux closure studies have been remarkably successful in achieving consistency between aerosol optical properties and surface broadband fluxes. Michalsky et al. [15] report that biases between measured and model flux are less than $2 \%$ for direct and diffuse components. On the other hand, broadband flux closure for the cloudy case is much more difficult because of the problem of determining cloud optical properties throughout the volume of atmosphere sensed by the surface radiometers and the concomitant potential of 3-D radiative transfer effects. Mindful of these problems, Turner et al. [1] and Comstock et al. [2] describe radiative flux closure efforts to assess the validity of retrieved values of $\tau$. In short, their flux closure schemes follow these steps: (1) take the retrieved value of $\tau$, and either assumed (or measured) optical properties of the cloud particles, (2) plug these values into a radiative transfer model, (3) calculate surface fluxes, and (4) compare calculated and observed surface fluxes. This scheme is followed approximately in [2] to show the relationship between retrieved $\tau$ values used to calculate surface fluxes and measured surface fluxes. They assumed that the ice particles possessed scattering properties of bullet-rosette crystals, and found that on a qualitative basis, measured and observed fluxes tracked each other reasonably well. Quantitative agreement was not nearly as good as for clear sky closure cases, illustrating the challenges associated with radiative flux closure in the presence of clouds.

\section{ALGORITHM EVALUATION}

\subsection{Evaluation Test Cases}

As noted above we choose data sets that permitted either a direct, independent determination of $\tau$ to be made, or that provided another means of independent verification, such as a radiative flux closure. The data sets used are listed in Table 1. The first data case, chosen to evaluate the algorithm under conditions of thin tropical cirrus, uses data from the ACRF TWP Darwin site, located in the northern coast of Australia. The data were obtained during the TWP-ICE campaign (http: //acrf-campaign.arm.gov/twpice/) that took place during early 2006. For several days during the campaign the Scaled Composites Proteus aircraft (http: //www.arm.gov/about/ 020206release.stm) executed spiral ascents and descents through the cloud layers allowing $\tau_{\text {est }}$ to be found. The second data set was chosen to evaluate the algorithm for midlatitude cirrus clouds. This data set was taken during the ARM Program's cloud intensive operational period (IOP) that took place during March 2000. A particular advantage of using this data set is that it has already been a subject of intense analysis of cloud optical depth retrieval algorithms [2], and this analysis includes an independent verification of the retrievals. 
Table 1. Data Sets Used for Algorithm Verification

\begin{tabular}{|c|c|c|c|}
\hline Name & Location (Site, Latitude, Longitude) & Time Period of Campaign & Range of $\boldsymbol{\mu}_{\boldsymbol{0}}$ \\
\hline \hline TWP-ICE & Darwin, Australia $\left(12.43^{\circ} \mathrm{S}, 130.89^{\circ} \mathrm{E}\right)$ & January, February 2006 & $0.53 \leq \mu_{0} \leq 0.87$ \\
\hline ARM Cloud IOP & Lamont, Oklahoma, USA $\left(\mathrm{N} 36^{\circ} 37^{\prime}, \mathrm{W} 97^{\circ} 30\right)^{\prime}$ & March 2000 & $0.39 \leq \mu_{0} \leq 0.75$ \\
\hline
\end{tabular}

During both of these campaigns, measurements were made of the total, direct, and diffuse components of the shortwave broadband irradiance using the usual suite of instruments: an Eppley Normal Incidence Pryheliometer (NIP) to measure the direct beam irradiances, and either an Eppley 8-48 Black \& White pyranometer (TWP-ICE) or an Eppley Precision Spectral Pyranometer (PSP, ARM Cloud IOP) to measure the diffuse irradiance. The PSP measurements were corrected for thermal offsets using the method of Younkin and Long [16]. Our algorithm requires as input the total irradiance, and for this quantity, we take the sum of the diffuse irradiance plus the direct beam irradiance multiplied by $\mu_{0}$.

The MFRSR was also operational during these campaigns. The MFRSR measures the diffuse, direct, and total components of the irradiances at six discrete wavelengths: $415,500,615,673$, and $870 \mathrm{~nm}$. We use the direct component of the irradiance at $415 \mathrm{~nm}$ to find $\tau$ using an algorithm similar in spirit to [17], in which apparent cloud optical depths obtained directly from the Beer-Lambert law are corrected by removing the effect of diffuse light scattered into the field of view of the detector $\left(3.3^{\circ}\right)$ by large cloud particles. The total optical depth derived directly from MFRSR data is $\tau_{\text {total }}=\tau_{\text {Rayleigh }}+\tau_{\text {aer }}+\tau_{\text {cloud,apparent, }}$, assuming no gaseous absorption, where $\tau_{\text {Rayleigh }}$ is the Rayleigh component. Subtracting this component and $\tau_{\text {aer }}$ from $\tau_{\text {total }}$ leaves the apparent cloud optical depth, $\tau_{\text {cloud,apparent }}$. This is an underestimate of the actual cloud optical depth because of the nonnegligible amount of forward scattering mentioned above.

Using Monte Carlo calculations the forward scattering contribution to the apparent cloud optical depth can be accounted for. The magnitude of this correction depends on the actual cloud optical depth, the size distribution, and cloud particle single scattering albedo. For the size distribution, we use that derived from TWP-ICE data, described in section. 4.2. Min et al. [17] suggest that the forward scattering corrections are not that sensitive to variations in the ice particle phase function, and this implies that the corrections derived using the TWP-ICE size distribution would be valid for other cases. Single scattering albedo was taken as a 1.0, because at $415 \mathrm{~nm}$ cloud particles do not absorb. From these calculations, we found that the correction to $\tau_{\text {cloud,apparent }}$ was very simple: for the solar zenith angles considered here, and for a wavelength of $415 \mathrm{~nm}$, the correction for ice clouds amounts to multiplying $\tau_{\text {cloud,apparent }}$ by 1.9 . When appropriate, we will show these optical depths, $\tau_{D B}\left(=1.9 \tau_{\text {cloud,apparent }}\right)$, termed "direct beam" (DB), as an aid for the evaluation of our algorithm.

\subsection{TWP-ICE}

We present results obtained from our algorithm for the day 25 January 2006, during the hours 1500 through 1700 LST, for this site LST $=$ UTC + 9.5. During this period, the high, thin cirrus was relatively uniform across the sky as shown from the image depicted in Fig. (2), taken from the Total Sky Imager (http: //www.arm.gov/instruments). The

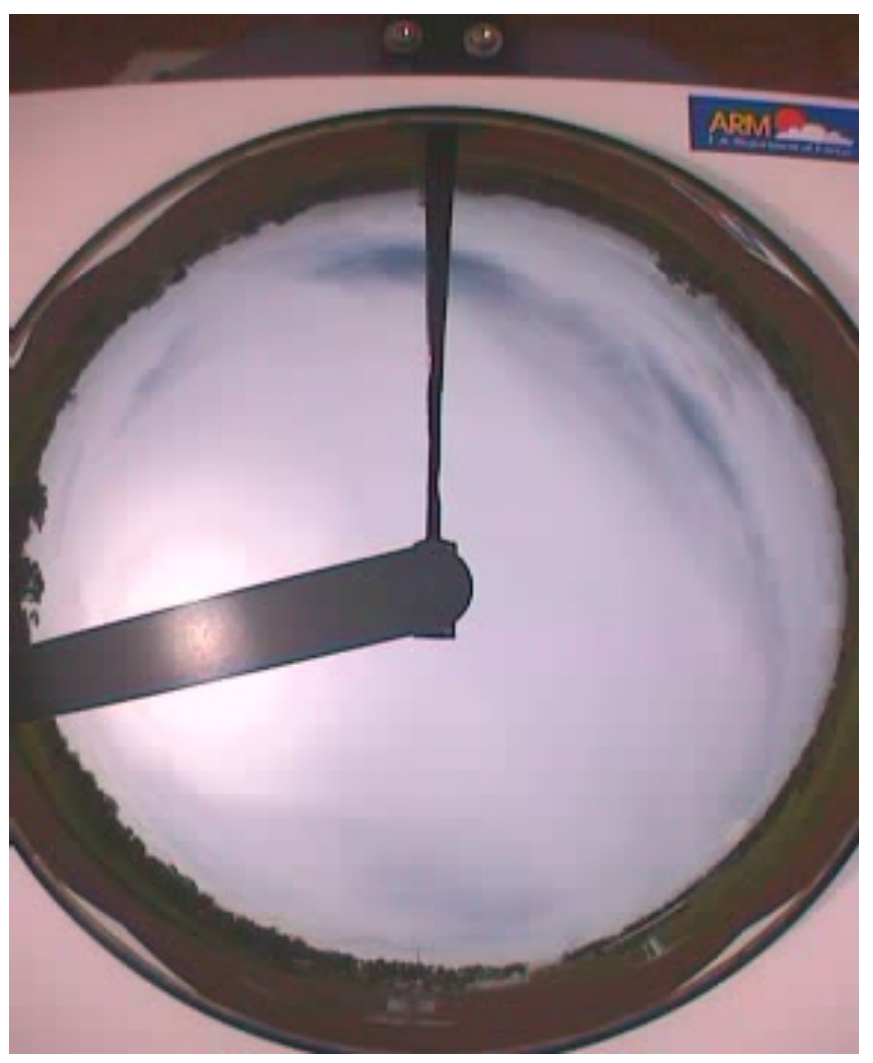

Fig. (2). Total sky imager snapshot taken at about 1600 LST, January 25,2006 , during the TWP-ICE campaign. The cirrus clouds in this scene are relatively optically thick. The fractional sky cover for this scene, from the routine of Long et al. [8], is 0.94 .

cloud sky cover was greater than 0.9 for the entire time period indicating that clouds filled the sky. This is essential because the plane-parallel assumption behind our simple algorithm is better satisfied than for more broken cloud scenes. During this day, at approximately 1650 LST, the Scaled Composites Proteus aircraft spiraled down through a cirrus cloud directly above the ACRF Darwin site, and measurements were made of the ice crystal shapes by the Stratton Park Engineering Company's (SPEC Inc.) Cloud Particle Imager (CPI), and of the ice crystal size distributions by Droplet Measurement Technology's (DMT) Cloud and Aerosol Spectrometer (CAPS) and the Cloud Imaging Probe 
(CIP). These size distributions were used to find cloud extinction as a function of height, $z$. Integration of the extinction over the depth of the cloud provides $\tau_{\text {est }}$. This quantity was calculated assuming various ice crystals habits (Yang et al. [18]) and the variation of habits resulted in a range of values for $\tau_{e s t}$ as will be illustrated in Fig. (3), discussed below.

To apply the algorithm we need values for $A$ and $g$. The area around the Darwin site consists of cleared tropical forests, city landscape, and ocean water. An area-average albedo appropriate for cirrus clouds with elevations between 10 and $15 \mathrm{~km}$ would have to account for the variation of albedo among these surfaces. According to Stull [19], urban land has an albedo of about 0.18 . Long et al. [20] have analyzed surface albedo measurements for several places in the Darwin area, including one site over ocean water in the Darwin harbor. The albedo at the Darwin ACRF site ranges from about 0.12 to 0.17 ; over the harbor, the albedo is variable, $0.06-0.16$, depending on wind speed and the condition of the water (e.g., murky water has a higher albedo). Using this information, we bracket the area-averaged albedo as lying between 0.06 and 0.18 . Given that the site lies inland of the ocean by several kilometers, and the clouds would tend to see more land than ocean, the area-averaged albedo probably lies towards the upper end of this range. Mindful of these considerations, we simply guess that the area-averaged albedo is 0.15 . We note that $\tau_{\text {empirical }} \propto 1 /(1-A)$ and therefore $\tau_{\text {empirical }}$ is not very sensitive to the exact value chosen for $A$ provided that the albedo values are small, which is typical for surfaces not covered by ice or snow.

The asymmetry parameter for ice crystals at visible wavelengths $(\lambda \approx 550 \mathrm{~nm})$ is generally less than that of spherical water droplets and regular hexagonal ice crystals [12]. Values reported in the literature from both theoretical calculations and measurements [12,18,21-24] range from a low of about 0.6 to a high of about 0.95 . These values depend on the ice crystal habit and the ice particle size distribution. In most applications, this information would not be available, and we assume an asymmetry parameter of 0.8 , which is about the midpoint of values reported in the literature. Clearly, because $\tau_{\text {empirical }} \propto 1 /(1-g)$, the results are sensitive to the exact value chosen for $g$. For example, a change in the assumed $g$ from 0.77 to 0.8 increases the calculated values by about $15 \%$.

Using these values for $A$ and $g$, calculations from our empirical algorithm and the DB values are shown in Fig. (3). The agreement between the DB values and the empirical algorithm is noteworthy and suggests, not surprisingly, that information regarding the cloud optical depth is found primarily in the direct beam irradiance. Also shown in this figure, as a vertical green line (at a time of about 16.8 LST), is the estimate of the first-principles optical depth, $\tau_{\text {est }}$, derived from size distributions measured by the CIP and CAS on the Proteus aircraft. The range of optical depth indicated by the green line is the range in values that stems from assuming different ice particle habits, mentioned above. This range

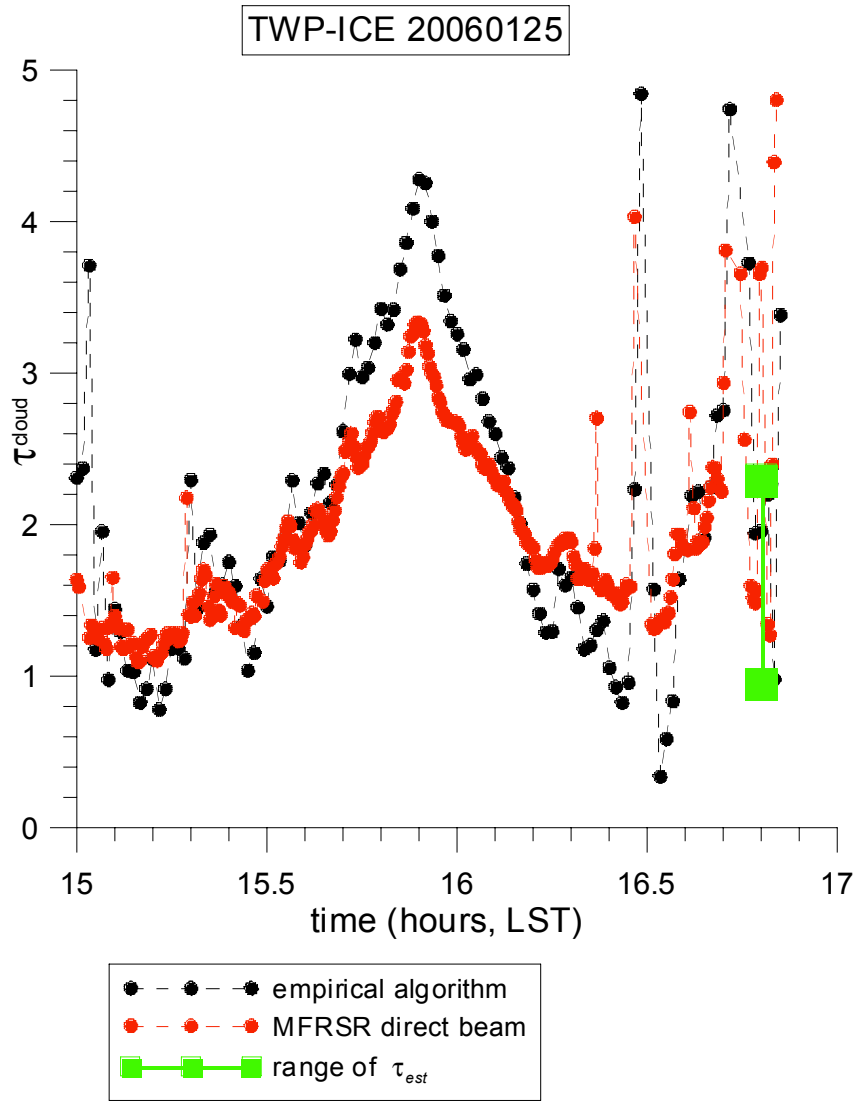

Fig. (3). Comparison of the output of the direct beam and empirical algorithms. Also shown by the vertical green line is the range of possible cloud optical depths derived from in situ sampling of ice particle size distributions. The range depends on the ice crystal habit chosen to convert size distribution information to extinction.

illustrates one of the difficulties of determining $\tau$ from surface measurements because the ice habit is generally unknown.

The inference of $\tau_{e s t}$ may be further affected by a problem related to the measurement of size distribution using instruments with shroud and inlets. McFarquhar et al. [14] provides evidence that the large ice crystals may have shattered on the inlet and shroud of the Cloud and Aerosol Spectrometer, artificially amplifying the numbers of ice crystals with maximum dimensions less than $50 \mu \mathrm{m}$. This can cause a significant overestimation of extinction (up to 106\%) and accordingly, $\tau_{e s t}$ shown in Fig. (3) may be an overestimation of the real $\tau$. Other uncertainties in measuring the size distributions include uncertainties induced from out-of-focus particles in the CIP that are resized following the Korolev and Isaac [25] algorithm and uncertainties in the concentration of particles with maximum dimensions between 50 and $125 \mu \mathrm{m}$ because of a poorly defined probe sample volume for such sized particles $[26,27]$ Without considering these factor, the uncertainty in $\tau$ because of unknown ice habit is about 1.3 units, ranging from 1 to 2.3. If we assume that ice shattering is contributing to an overestimation of $\tau_{e s t}$, both limits to this range would be lower. As indicated in Fig. (3), the empirical and the DB values of $\tau$ lie approximately within the range of likely $\tau_{e s t}$ values. However, if $\tau_{e s t}$ is too large by a factor of 
two because of the ice shattering problem, then the upper bound of the range is about 1.2, and the empirical and DB values would lie slightly above this bound.

\subsection{ARM Cloud IOP}

During the month of March 2000, a cloud intensive operational period (IOP) was held at the ACRF SGP facility. This IOP included aircraft flights over the site and the deployment of a plethora of instruments, allowing $\tau$ to be estimated using the data from these instruments and their associated algorithms. Abundant single layer cirrus clouds were present over the SGP site on 9 March 2000 during the afternoon. These clouds, as seen in Fig. (4) that shows a cloud image from the Whole Sky Imager (www.arm.gov), are not horizontally homogenous and the sky at this time contains significant clear patches. The lack of horizontal homogeneity presents a challenge to our algorithm because of the planeparallel assumption required by its derivation.

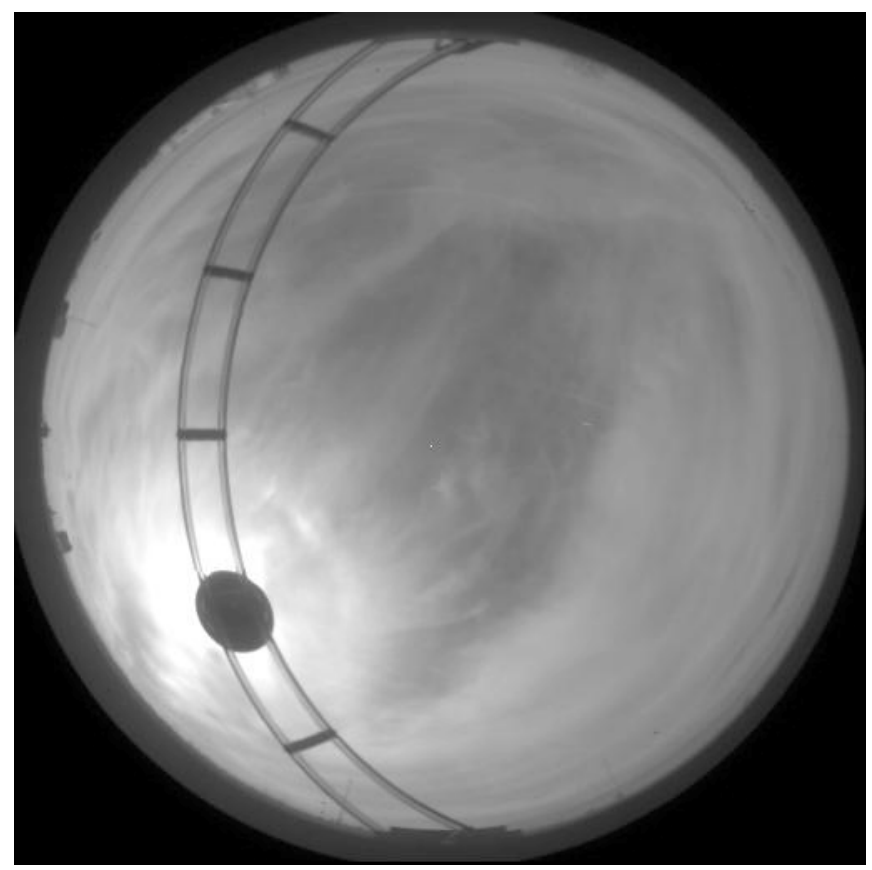

Fig. (4). Whole sky imager view of the sky for March 9, 2000, at time 2130 UT (1530 LST). The fractional sky cover at this time was 0.90 .

We examine a time period that is identical to the period studied by Comstock et al. [2], 1900 to 2230 UTC. (For the SGP site, LST $=$ UTC -6 , so the time period under consideration occurs in the afternoon). For this time interval, Comstock et al. evaluated retrieval algorithms designed to find cloud microphysical properties from ground-based instruments. These zenith-pointing instruments include cloud radars, regular and Raman lidars, and an Atmospheric Emitted Radiance Interferometer (AERI). Comstock et al. show a time series of an averaged value of $\tau$ (henceforth called $\tau_{\text {ave }}$ ), where the average is taken over all the algorithms. Aircraft and satellite overpasses permitted the algorithms to be compared with independent data including in situ samples of ice water path (IWP) and satellite retrievals of $\tau$. For those algorithms that could retrieve IWP, comparisons between the retrieved and in situ IWP revealed reasonable agreement, considering the many difficulties facing independent verification of retrieved $\tau$ and IWP. A flux closure study shows that, when using $\tau_{\text {ave }}$ as input to a radiative transfer model, measured and modeled downwelling shortwave fluxes track each another qualitatively, but the agreement is nowhere near exact. This comparison is difficult because the 3-D radiative transfer that occurs in the broken overcast sky is difficult to simulate correctly, as noted in Comstock et al.

These validation exercises suggest that $\tau_{a v e}$ is at least a "state of the art" estimate of the true value of $\tau$, and accordingly we take $\tau_{\text {ave }}$ as our standard of comparison. Fig. (5) shows the $\tau_{\text {ave }}, \tau_{\text {empirical }}$, and $\tau_{D B}$ from the direct beam measurements of the MFRSR. Note the logarithmic scale for the ordinate of this figure. As mentioned at the end of section 2 the empirical algorithm may not work well when the cloud optical depth is very low and can even produce values of $\tau_{\text {empirical }}$ that are negative. Negative values were produced for some of the time during the test case period, particularly around 22.2 UTC. For a small interval about this time, the algorithm simply did not work, as expected, because of the small cloud optical depth. If we do not consider this period, the empirical algorithm compares favorably with $\tau_{a v e}$ for a significant part of the time period considered here, over a range of $\tau$ from about 0.1 to about 5.0. The good agreement for lower optical depth is a little surprising given the intrinsic uncertainty in optical thickness for very thin clouds. Comparing the median values of $\tau_{\text {empirical }}$ and $\tau_{\text {ave }}$ using a set of points where both these values exist (i. e., we must exclude the time period where the algorithm produces negative $\tau_{\text {empirical }}$ values), we find that the medians are 0.81 for $\tau_{\text {ave }}$ and 0.79 for the empirical method. Although the small difference of only 0.02 is likely to be fortuitous, it suggests that the empirical method is able to approximately capture some bulk statistics of the cloud optical depth field. Additionally, a comparison of the difference in optical depths between $\tau_{\text {empirical }}$ and $\tau_{\text {ave }}$, as a function of $\mu_{0,}$ revealed no obvious bias with respect to $\mu_{0}$.

During some time intervals, for example 1900 to 1930 UTC, the $\tau_{\text {empirical }}$ and $\tau_{\text {ave }}$ values diverge. This could be a consequence of $\tau_{\text {ave }}$ being inferred from vertically pointing, narrow field of view instruments and thus representing only the portion of the cloud directly above the site, while the empirical algorithm uses the total component of the irradiance, a hemispherical quantity. Moreover, for very low cloud optical depths, the direct beam component composes the largest part (on the order of $75 \%$ ) of the total component of the downwelling irradiance for the solar zenith angles considered here, and the empirical algorithm, like the DB method, would therefore be most sensitive to clouds between the surface and the sun. That this conjecture is true can be seen by comparing the empirical and DB methods: they provide similar results over most of the time domain of interest. Therefore some of the discrepancy seen between the time series of $\tau_{\text {ave }}$ and $\tau_{\text {empirical }}$ is probably caused by different field of views. 


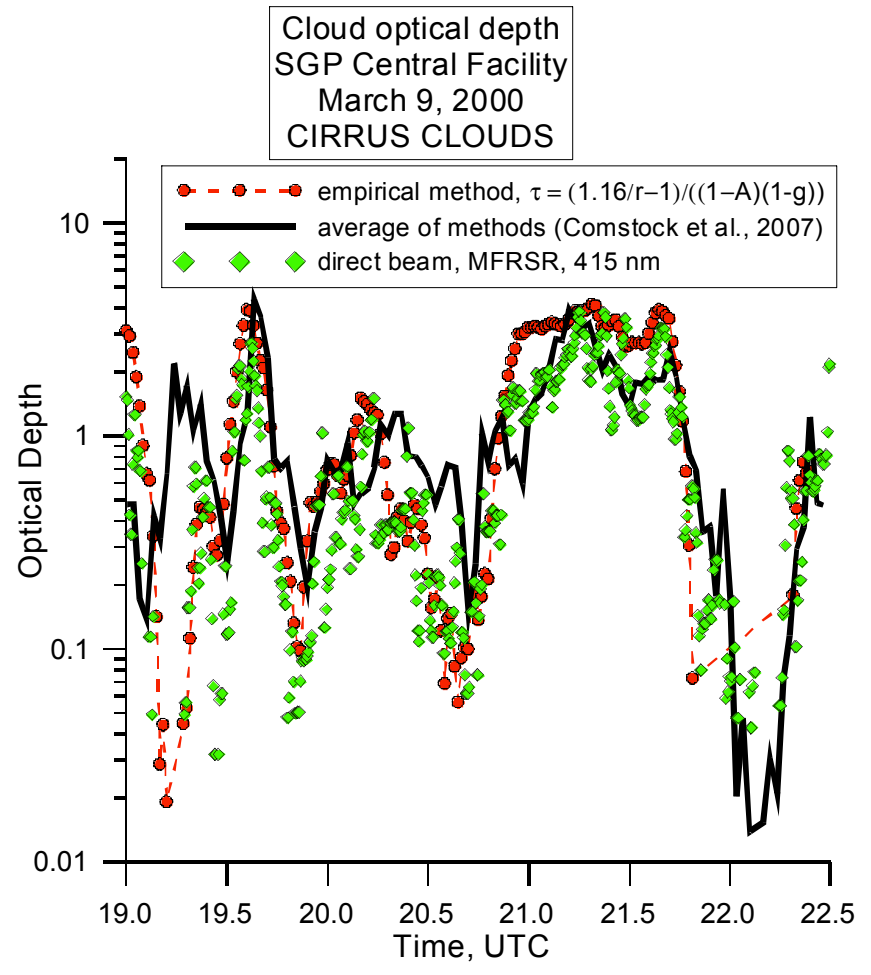

Fig. (5). Comparison of cloud optical depths derived from the empirical algorithm and the DB method on March 9, 2000. Also shown by the thick black line is $\tau_{\text {ave }}$, the average optical depth of many retrievals as described in [2].

\subsection{Comparison to Long-Term Lidar Retrievals}

The primary purpose of the comparisons discussed above is to examine how well the algorithm performs when independent verification of cloud optical depth is available. These comparisons are essential, but the time periods over which they apply are too short to permit a thorough evaluation of the algorithm. As mentioned above, the apparently good agreement witnessed in the SGP case is likely to be fortuitous, and not a true indicator of algorithm performance. Therefore, we wish to evaluate the algorithm over a longer period of time.

We present below a comparison of the $\tau_{\text {empirical }}$ with cloud optical depths obtained from the Raman lidar at the ARCF SGP site, labeled $\tau_{\text {lidar }}$. The algorithm that converts the lidar backscatter data to $\tau_{\text {lidar }}$ has been described in [28]. The comparison extends over a three-year period (1998, 1999, and 2000) but during most of this period, there was little independent verification of $\tau_{\text {lidar }}$ except during the ARM Cloud IOP, during which the average value of $\tau_{\text {lidar }}$ was 0.45 , while $\tau_{\text {ave }}$ was 1.08 .

Because point-by-point comparisons are noisy, in part because the lidar is a zenith-pointing instrument and our algorithm is applicable to a full sky view, we show the distributions of $\tau_{\text {empirical }}$ and $\tau_{\text {lidar }}$. Additionally, there are numerous occasions where the lidar only sees a thin cloud in its narrow field of view, but the sky is filled with other, optically thicker clouds, which are sensed by our method. To eliminate most of these cases, we require that the cloud sky cover is greater than $95 \%$ (as before), and we also require that the cloud transmission, defined as $T / C$, is greater than $80 \%$. This last restriction tends to limit the comparison to cases when both the lidar and broadband sensors are seeing thin clouds because the cloud transmission is so large.

When restricted in this manner, there are only 124 tenminute cases over the three years that meet the criteria described above. A major reason for the small number of cases is that the lidar retrievals are most successful during the night, whereas our algorithm can be applied only during the day. The distributions are formed by binning the optical depths in bins of one unit of width, 0 to 1,1 to 2 , etc. The distributions so derived are shown in Fig. (6), and show the same general trend: many thin clouds in the bin 0 to 1 , with fewer clouds as the optical depth increases. Beyond an optical thickness of four, the lidar attenuation through a cloud is so large that the backscattered signal is too small to allow retrievals. Therefore, the distributions drop to virtually zero for $\tau_{\text {lidar }}$ greater than four. The difference between the lidarderived and empirically derived distribution in the smallest optical depth bin $(0-1)$ may be caused by the empirical method's difficulties predicting small optical depths. (Recall that we do not expect this method to work well for these situations).

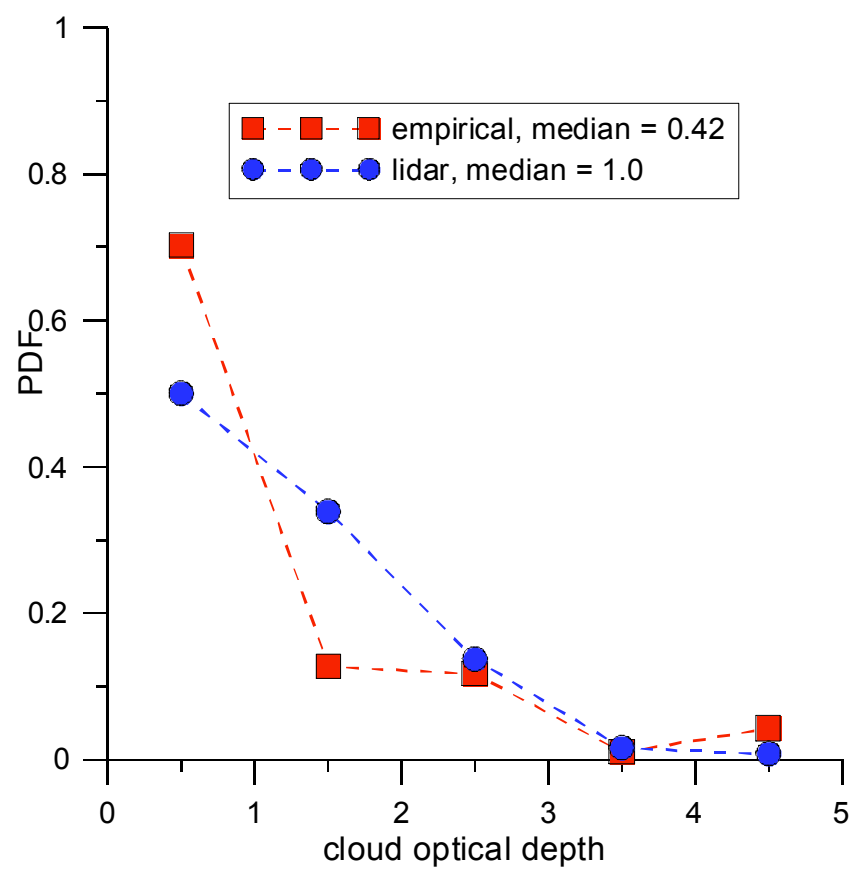

Fig. (6). Distribution of thin cloud optical depth derived from lidar data, and the empirical method, for the years 1999, 2000, and 2001 and the ACRF SGP site.

The median values of $\tau_{\text {empirical }}$ and $\tau_{\text {lidar }}$ over all cases are 0.42 and 1.0, respectively. Because of the lack of independent verification, we cannot say which of these median values is closer to the median of the actual optical depth distribution; all we can state is that the median values agree to about 0.6 .

\subsection{Ice or Liquid Water?}

The algorithm yields different values of $\tau_{\text {empirical }}$ depending upon whether the clouds are assumed to be ice or liquid 
water. The type of cloud is represented in the calculation by the choice of $g$, which we recommend be set to 0.87 and 0.8 , for liquid water and ice clouds respectively. An intermediate value could be chosen for mixed phase clouds, but we have not examined this case explicitly. The algorithm relies on broadband measurements alone, and if these are the only measurements available, one might wonder how to distinguish between ice and liquid water clouds. Based on the experience described above, where we obtained comparable agreement between $\tau_{\text {empirical }}$ and $\tau_{\text {lidar }}$ using cloud transmission as a crude discriminator between ice and liquid clouds, we suggest using a value of $T / C$ as 0.8 to distinguish between the two types of clouds. In other words, we assume that thin clouds are only ice clouds. However, if other instrumentation is deployed at a site, such as an infrared thermometer (IRT) that senses cloud base temperature, then the process of distinguishing between ice and water clouds could be markedly improved.

\section{CONCLUSIONS}

We describe here the development and testing of an algorithm for finding cloud optical depth from surface broadband irradiance measurements. The derivation of this algorithm is dependent on the assumptions of horizontal homogeneous and optically thick clouds. Although the second of these assumptions, by definition, will never be true for thin clouds, and the first will rarely be true, we nevertheless apply the method to the case of optically thin ice clouds, and find that the method works surprising well. The algorithm is evaluated by comparing our results with those from other retrievals and independent data. The evaluation is made much easier by the use of ice cloud data sets that have been studied thoroughly as exemplified in the study of Comstock et al. [2] and the TWP-ICE campaign The study of Comstock et al. compares various algorithms and attempts algorithm verification using independent data and flux closure studies. For the TWP-ICE campaign, independent verification is available from aircraft sampling of ice cloud properties.

When applied to ice clouds, the algorithm may not work well when the optical depth is low ( 0.1 or less), but seems to work reasonably well, vis-à-vis other algorithms, for optical depths from about 0.1 to 5.0 , at least for the cases examined here. We could not test the algorithm for larger values of icecloud optical depth because we did not have data that includes ice clouds with optical depths that large. For the limited cases we examined here, the empirical cloud optical depth and an independent determination of the optical depth agreed to about 1 unit for the TWP-ICE case. For the SGP case, the empirical optical depth roughly tracks an average optical depth derived from other methods. In particular, for a time series consisting of points when: (1) values of both $\tau_{\text {ave }}$ and $\tau_{\text {empirical }}$ are available, and (2) $\tau_{\text {empirical }}$ is greater than zero, the median values obtained from these two methods are $0.79\left(\tau_{\text {ave }}\right)$ and $0.81\left(\tau_{\text {empirical }}\right)$-- a difference of only 0.02 units. Such close agreement, however, is probably fortuitous and unlikely to represent the performance of the algorithm, thus suggesting the need for further evaluation. To achieve this end we compare distributions of $\tau_{\text {empirical }}$ to distributions of optical depths derived from lidar data at the ACRF SGP site over a three-year period. The median values of these distributions over all cases are 0.42 and 1.0 , for $\tau_{\text {empirical }}$ and $\tau_{\text {lidar }}$ respectively, a difference of about 0.5 . We remark, however, that because of the lack of independent verification over most of the three-year period, we cannot say which of these median values most faithfully represents the actual optical depth.

The algorithm would not be expected to work well for isolated cirrus clouds because these cases contradict the assumption of plane parallel conditions. Fortunately, these cases can be eliminated using Long et al.'s [8] fractional sky cover routine that can detect cases where the sky is mostly clear. For smaller fractional sky covers, the DB method could be used to infer $\tau$, but this method would only provide the optical depth of the clouds that are situated in the path between the sun and earth.

Because of this method's simplicity and its reliance on standard shortwave irradiance measurements, it may be applied to estimate $\tau$ for both optically thin and optically thick clouds at the many worldwide locations where such measurements exist. However, when only broadband measurements are available, it becomes problematic to distinguish between thin ice clouds and thin liquid water clouds. This problem can be mitigated by employing an infrared thermometer (or similar instruments) to detect the cloud base temperature. Absent such an instrument, we recommend using the cloud transmission, $T / C$, as a crude discriminator between ice and liquid clouds, with values of $T / C>0.8$ suggesting the presence of ice clouds.

\section{ACKNOWLEDGEMENTS}

This research was sponsored by the U. S. Department of Energy's Atmospheric Radiation Measurement Program (ARM) under Contract DE-AC06-76RLO 1830 at Pacific Northwest National Laboratory. Pacific Northwest National Laboratory is operated by Battelle for the U.S. Department of Energy. The authors wish to thank Dr. Chris Doran for his comments regarding this work, and Nancy Burleigh and Ruth Keefe for their help with this manuscript.

\section{REFERENCES}

[1] Turner DD, Vogelmann AM, Austin RT, et al. Thin liquid water clouds - Their importance and our challenge. Bull Am Meteor Soc 2007; 88(2): 177-190.

[2] Comstock JM, d'Entremont R, DeSlover D, et al. An intercomparison of microphysical retrieval algorithms for upper-tropospheric ice clouds. Bull Am Meteor Soc 2007; 88(2): 191-204.

[3] Barnard JC, Long CN. A simple empirical equation to calculate cloud optical thickness using shortwave broadband measurements. J Appl Meteor 2004; 43(7): 1057-1066.

[4] Long CN, Ackerman TP. Identification of clear skies from broadband pyranometer measurements and calculation of downwelling shortwave cloud effects. J Geophys Res 2000; 105(D12): 15609-15626.

[5] Dong XQ, Ackerman TP, Clothiaux EE, Pilewskie P, Han Y. Microphysical and radiative properties of boundary layer stratiform clouds deduced from ground-based measurements. J Geophys Res 1997; 102(D20): 23829-23843.

[6] Min QL, Harrison LC. Cloud properties derived from surface MFRSR measurements and comparison with GOES results at the ARM SGP site. Geophys Res Lett 1996; 23(13): 1641-1644. 
[7] Harrison L, Michalsky J, Berndt J. Automated multifilter rotating shadow-band radiometer - an instrument for optical depth and radiation measurements. Appl Opt 1994; 33(22): 5118-5125.

[8] Long CN, Ackerman TP, Gaustad KL, Cole JNS. Estimation of fractional sky cover from broadband shortwave radiometer measurements. J Geophys Res 2006; 111, doi: 10.1029/2005JD006475.

[9] Shettle EP, Weinman JA. Transfer of solar irradiance through inhomogeneous turbid atmospheres evaluated by eddingtons approximation. J Atmos Sci 1970; 27(7): 1048-1055.

[10] Hu YX, Stamnes K. An Accurate parameterization of the radiative properties of water clouds suitable for use in climate models. J Clim 1993; 6(4): 728-742.

[11] Goody RM, Yung YL. Atmospheric Radiation, Theoretical Basis. Oxford University Press, Oxford, New York, 1989.

[12] Liou KN. Radiation and Cloud Processes in the Atmosphere. Oxford University Press, Oxford, New York, 1992.

[13] Heymsfield AJ, Bansemer A, Field PR, et al. Observations and parameterizations of particle size distributions in deep tropical cirrus and stratiform precipitating clouds: Results from in situ observations in TRMM field campaigns. J Atmos Sci 2002; 59(24): 3457-3491.

[14] McFarquhar GM, Um J, Freer M, Baumgardner D, Kok GL, Mace G. Importance of small ice crystals to cirrus properties: Observations from the Tropical Warm Pool International Cloud Experiment (TWP-ICE). Geophys Res Lett 2007; 34, doi: 10.1029/2007 GL0 29865.

[15] Michalsky JJ, Anderson GP, Barnard J, et al. Shortwave radiative closure studies for clear skies during the atmospheric radiation measurement 2003 aerosol intensive observation period. J Geophys Res 2006; 111, doi: 10.1029/2005JD006341.

[16] Younkin K, Long CN. Improved correction of IR loss in diffuse shortwave measurements: An ARM Value Added Product. ARM TR-009, 2004; http: //www.arm.gov/publications/techreports.stm.

[17] Min QL, Joseph E, Duan MZ. Retrievals of thin cloud optical depth from a multifilter rotating shadowband radiometer. J Geophys Res 2004; 109, doi: 10.1023/2003JD003964.
[18] Yang P, Liou KN, Wyser K, Mitchell D. Parameterization of the scattering and absorption properties of individual ice crystals. J Geophys Res 2000; 105(D4): 4699-4718.

[19] Stull R. An Introduction to Boundary Layer Meteorology. Kluwer Academic Press, Dordrecht, Boston, 1991.

[20] Long CN, Mather JH, Tapper N, Beringer J, Atkinson B. Surface radiation analysis from TWP-ICE. Proceedings of Sixteenth ARM Science Team Meeting, Albuquerque, New Mexico, March 27-31, 2006, http: //www.arm.gov/publications/proceedings/conf16/extended_abs/long_cn1.pdf.

[21] Gerber H, Takano Y, Garrett TJ, Hobbs PV. Nephelometer measurements of the asymmetry parameter, volume extinction coefficient, and backscatter ratio in Arctic clouds. J Atmos Sci 2000; 57(18): 3021-3034.

[22] Ulanowski Z, Hesse E, Kaye PH, Baran AJ. Light scattering by complex ice-analogue crystals. J Quant Spectrosc Radiat Transfer 2006; 100(1-3): 382-392.

[23] Schmitt CG, Iaquinta J, Heymsfield AJ. The asymmetry parameter of cirrus clouds composed of hollow bullet rosette-shaped ice crystals from ray-tracing calculations. J Appl Meteor Climatol 2006; 45(7): 973-981.

[24] Um J, McFarquhar GM. Single-scattering properties of aggregates of bullet rosettes in cirrus. J Appl Meteor Climatol 2007; 46(6): 757-775.

[25] Korolev A, Isaac GA. Relative humidity in liquid, mixed-phase, and ice clouds. J Atmos Sci 2006; 63(11): 2865-2880.

[26] Baumgardner D, Korolev A. Airspeed corrections for optical array probe sample volumes. J Atmos Ocean Technol 1997; 14(5): 12241229.

[27] Strapp JW, Albers F, Reuter A, et al. Laboratory measurements of the response of a PMS OAP-2DC. J Atmos Ocean Technol 2001; 18(7): 1150-1170.

[28] Comstock JM, Sassen K. Retrieval of cirrus cloud radiative and backscattering properties using combined lidar and infrared radiometer (LIRAD) measurements. J Atmos Ocean Technol 2001; 18(10): 1658-1673. 